\title{
Resistance patterns and integron cassette arrays of Enterobacter cloacae complex strains of human origin
}

\begin{abstract}
Correspondence
Joanna Mokracka

amok@amu.edu.pl
\end{abstract}

Received 23 November 2010

Accepted 15 February 2011

\author{
Joanna Mokracka, Ryszard Koczura, Konrad Pawłowski \\ and Adam Kaznowski
}

Department of Microbiology, Faculty of Biology, A. Mickiewicz University, Poznań, Poland

The aim of this research was to analyse the resistance patterns and characterize the distribution and genetic content of resistance integrons within Enterobacter cloacae complex strains originating from hospitalized patients. The strains were included in the $E$. cloacae complex study following sequence analysis of the $h s p 60$ gene. The determination of resistance towards eight classes of antimicrobials was followed by PCR detection of integrons and analyses of the size and sequences of their variable parts. The majority of 69 clinical strains of the $E$. cloacae complex were identified as Enterobacter hormaechei. They were isolated from a variety of samples, including urine, wounds, blood and stools. The remaining isolates belonged to E. cloacae clusters III and IV, E. cloacae subsp. cloacae and Enterobacter kobei. Fifty-two isolates (75.4\%) were resistant to more than three unrelated antibiotics. The resistance for each antibiotic, except imipenem, was significantly associated with the presence of integrons. Class 1 integrons were detected in $55 \%$ of isolates: $63.3 \%$ of ' $E$. hormaechei subsp. steigerwaltii', $50 \%$ of $E$. cloacae cluster III, $40 \%$ of ' $E$. hormaechei subsp. oharae', $33 \%$ belonging to $E$. cloacae cluster IV and $20 \%$ of ' $E$. hormaechei subsp. hormaechei' were int/1-positive. All of the integrons were located on transferable genetic elements. The transferred resistance primarily included that to aminoglycosides, ticarcillin, piperacillin, sulfamethoxazole, trimethoprim and tetracycline.

Sequence analysis of the variable regions of integrons identified two groups of genes: those encoding aminoglycoside adenylotransferases responsible for resistance to aminoglycosides, and $d f r$ cassettes conferring resistance to trimethoprim. Integrons of the $E$. cloacae complex showed limited variability of genes encoding resistance to therapeutics and were stable in structure with the following cassette arrays: dfrA12-orfF-aadA2, aadB-aadA2, dfrA1-aadA1 and aacA4-aadA1. Hospital-dependent differences in type and arrays of gene cassettes were observed, which seemed to be conserved and not liable to changes.

\section{INTRODUCTION}

Bacteria of the Enterobacter cloacae complex are Gramnegative, chemo-organotrophic, facultatively anaerobic rods or coccobacilli. Recently, the importance of members of the E. cloacae complex as nosocomial pathogens has been highlighted, and strains belonging to this species are encountered among the most-often-isolated strains able to cause severe opportunistic infections in hospitalized and debilitated patients, especially in intensive care units. They cause pneumonia and urinary tract, wound, skin and soft tissue, ophthalmic and bloodstream infections (Fraser et al.,

Abbreviations: CS, conserved segment; ERIC, enterobacterial repetitive intergenic consensus.

A dendrogram showing the genetic relatedness of the 69 clinical strains of the E. cloacae complex is available with the online version of this paper.
2008). By using commercial biochemical kits, they are routinely identified as 'E. cloacae'. The complex is, however, heterogeneous. According to the sequence of the hsp 60 gene, it has been divided into 12 genetic clusters: Enterobacter asburiae, E. cloacae subsp. cloacae, E. cloacae subsp. dissolvens, E. cloacae cluster III, E. cloacae cluster IV, E. cloacae cluster IX, 'Enterobacter hormaechei subsp. hormaechei, 'E. hormaechei subsp. oharae', 'E. hormaechei subsp. steigerwaltii, Enterobacter kobei, Enterobacter ludwigii and Enterobacter nimipressuralis (Hoffmann \& Roggenkamp, 2003). The members of the E. cloacae complex differ in pathogenicity towards humans. ' $E$. hormaechei subsp. steigerwaltii', 'E. hormaechei subsp. oharae' and E. cloacae cluster III are the species most frequently recovered from clinical specimens (Hoffmann et al., 2005; Morand et al., 2009). Moreover, E. hormaechei was reported to have caused an epidemic outbreak (Paauw et al., 2009). 
Bacteria of the E. cloacae complex are becoming increasingly prevalent nosocomial pathogens, probably due to higher levels of resistance to disinfectants and antimicrobial agents in comparison with other members of the Enterobacteriaceae (Paauw et al., 2008). E. cloacae is naturally resistant to ampicillin, cephalotin and other older cephalosporins, and cefoxitin. A growing number of resistant Enterobacter species isolates, particularly those resistant to broad-spectrum cephalosporins, has been observed worldwide (Kang et al., 2004). Therapy for Enterobacter species infections is often complicated by multiple antibiotic resistance, a phenomenon that leads to higher morbidity and mortality in infections caused by strains of Enterobacteriaceae and substantially increases treatment costs (Daikos et al., 2007; Nijssen et al., 2005; Norrby, 2005). Antimicrobial resistance may develop through mutations in chromosomal DNA or horizontal gene transfer including acquisition of plasmids or transposons carrying resistance determinants often assembled in platforms called integrons (Hall \& Collis, 1995). The integron consists of an integrase gene of the tyrosine recombinase family, a primary recombination site called attI and a $\mathrm{P}_{\mathrm{C}}$ promoter that directs transcription of the captured genes. Five classes of resistance integrons have been defined based on the polymorphism of the integrase genes. Three classes of integrons are responsible for multidrug resistance, with class 1 being most ubiquitous among resistant bacteria, and are considered to play the main role in the emergence and wide dissemination of resistance genes (Fluit \& Schmitz, 2004; Mazel, 2006). Moreover, integron-mediated resistance can be silenced in a bacterial population at no biological cost, which means that resistant bacteria persist even if the selective pressure imposed by antibiotic usage is reduced (Andersson \& Hughes, 2010).

The prevalence of multidrug resistance among isolates of the E. cloacae complex in human infections is rising. Therefore, the mechanisms of resistance, role of appropriate antimicrobial therapy and effect of specific antimicrobial usage must be taken into consideration.

The aim of this research was to analyse the resistance patterns and characterize the distribution and genetic content of resistance integrons within the E. cloacae complex originating from hospitalized patients.

\section{METHODS}

Clinical specimens. Sixty-nine unique strains of E. cloacae were collected from December 2006 to May 2008. They were obtained from specimens of inpatients at two Polish hospitals located $300 \mathrm{~km}$ away from each other. The organisms were grown on MacConkey Agar No. 3 (Oxoid) and identified using API 20E (bioMérieux) as E. cloacae. They were isolated from urine (30 strains), blood (ten strains), wounds (seven strains), catheters (three strains), anus swabs (four strains), aspirate (four strains), peritoneal cavity fluid (two strains), ulceration (two strains) and bronchoalveolar lavage, abscess, ear, eye, throat, gall bladder and faeces (a single strain from each).
Molecular identification of strains by $\boldsymbol{h s p} 60$ sequence analysis. The strains were further identified by sequence analysis of the $h s p 60$ gene. Bacterial DNA was isolated using a Novabeads Bacterial Genomic DNA kit (Novazym). Hi-Fi Taq polymerase and other PCR reagents were also purchased from Novazym. Primers Hsp60-F and Hsp60-R were used to amplify a portion of $h s p 60$ by PCR according to Morand et al. (2009). The PCR products were purified using ExoSAP-IT (Affymetrix) and sequenced in a 3130xl Genetic Analyzer (Applied Biosystems). A $272 \mathrm{nt}$ fragment of the sequence was compared with the sequences of type and reference strains using CLUSTAL W and the neighbour-joining method (Morand et al., 2009). The hsp60 sequences of the following type and reference strains were used: E. cloacae subsp. cloacae ATCC $13047^{\mathrm{T}}$, E. cloacae subsp. dissolvens ATCC $23373^{\mathrm{T}}$, E. cloacae cluster III EN114, E. cloacae cluster IV EN117, E. cloacae cluster IX EN25, E. asburiae ATCC $35953^{\mathrm{T}}$, E. kobei ATCC BAA-260' ${ }^{\mathrm{T}}$ E. ludwigii $\mathrm{EN}-119^{\mathrm{T}}$, ' $E$. hormaechei subsp. oharae' $\mathrm{EN}-314^{\mathrm{T}}$, 'E. hormaechei subsp. hormaechei' ATCC $49162^{\mathrm{T}}$, 'E. hormaechei subsp. steigerwaltii' CIP $108489^{\mathrm{T}}$, E. nimipressuralis ATCC $9912^{\mathrm{T}}$, Enterobacter cancerogenus ATCC $33241^{\mathrm{T}}$, Enterobacter amnigenus ATCC $33072^{\mathrm{T}}$, Enterobacter cowanii CIP $107300^{\mathrm{T}}$, Enterobacter gergoviae ATCC $33028^{\mathrm{T}}$, Enterobacter pyrinus ATCC $49851^{\mathrm{T}}$ and Enterobacter sakazakii ATCC $29544^{\mathrm{T}}$ (Hoffmann \& Roggenkamp, 2003; Morand et al., 2009).

Enterobacterial repetitive intergenic consensus (ERIC)-PCR typing. The ERIC-PCR method utilizes primers complementary to enterobacterial repetitive intergenic consensus sequences of genomic DNA. A PCR using primers ERIC 1 and ERIC 2 was carried out according to Versalovic et al. (1991). Computer analysis of electrophoretic patterns was carried out using GelCompar II version 3.5 software (Applied Maths): similarities between fingerprints were calculated with the Dice coefficient and cluster analysis was performed by UPGMA.

Antimicrobial susceptibility. The susceptibility to 15 antibiotics representing eight classes was determined according to the standard disc diffusion method recommended by the Clinical and Laboratory Standards Institute (CLSI, 2009). The antimicrobials were: amikacin, tobramycin, netilmicin, gentamicin, ticarcillin, ciprofloxacin, tetracycline, cefotaxime, ceftazidime, sulfamethoxazole, co-trimoxazole, piperacillin, piperacillin + tazobactam, chloramphenicol and imipenem. Production of extended-spectrum $\beta$-lactamases was checked by a double-disc synergy test with ceftazidime, cefotaxime and amoxicillin/clavulanic acid. The production of AmpC $\beta$-lactamases was checked by a three-dimensional assay according to Coudron et al. (2000). Klebsiella pneumoniae carbapenemases were detected by disc test with phenylboronic acid and imipenem (Tsakris et al., 2009). Metallo- $\beta$-lactamase production was checked by an EDTA-disc synergy test (Lee et al., 2001). All antibiotic discs were provided by Oxoid.

Multiplex PCR analyses for identification of integron integrase genes. PCR amplifications were performed in a $50 \mu$ volume with $5 \mu \mathrm{l} 10 \times$ PCR buffer with $\mathrm{NH}_{4}\left(\mathrm{SO}_{4}\right)_{2}, 0.6 \mu \mathrm{M}$ each primer, $200 \mu \mathrm{M}$ dNTP mix, $2.5 \mathrm{mM} \mathrm{MgCl}_{2}$, $2 \mathrm{U}$ Hi-Fi Taq polymerase and $200 \mathrm{ng}$ genomic DNA. Amplification involved initial denaturation at $94{ }^{\circ} \mathrm{C}$ for $5 \mathrm{~min}$, followed by 30 cycles of denaturation at $94{ }^{\circ} \mathrm{C}$ for $1 \mathrm{~min}$, annealing at $59{ }^{\circ} \mathrm{C}$ for $1 \mathrm{~min}$ and extension at $72{ }^{\circ} \mathrm{C}$ for $1 \mathrm{~min}$, with a final extension step at $72{ }^{\circ} \mathrm{C}$ for $8 \mathrm{~min}$. The sequences of the primers targeting the intI1, intI2 and intI3 genes were those recommended by Dillon et al. (2005).

Analysis of the variable region of class 1 integron by conserved segment (CS)-PCR. Sequences of primers complementary to the $5^{\prime}$ and $3^{\prime}$-conserved regions of class 1 integrons have been published elsewhere (Lévesque et al., 1995). PCR amplification was conducted as follows: initial denaturation at $94{ }^{\circ} \mathrm{C}$ for $5 \mathrm{~min}$, followed by 30 cycles 
of $94{ }^{\circ} \mathrm{C}$ for $1 \mathrm{~min}, 59{ }^{\circ} \mathrm{C}$ for $1 \mathrm{~min}$ and $72{ }^{\circ} \mathrm{C}$ for $5 \mathrm{~min}$, with a final elongation at $72{ }^{\circ} \mathrm{C}$ for $8 \mathrm{~min}$.

All PCRs were performed in a C1000 Thermal Cycler (Bio-Rad) and the products were separated in a $1.5 \%$ agarose gel (Prona). The molecular masses of the PCR products were determined by Bio-Capt version 99.04 software (Vilber Lourmat). All experiments were carried out in triplicate.

CS-PCR products were purified with ExoSAP-IT and sequenced. When two amplicons were present, the PCR products were separated by electrophoresis and purified using a QIAquick Gel Extraction kit (Qiagen). Some of the products were cloned using the pGEM-T Easy vector (Promega). Sequence data were analysed with DNA Baser (Heracle Software) and aligned with available GenBank data using BLAST.

Conjugation assay. Strains positive for the intI gene were examined for the ability to transfer resistance by conjugation. The recipient strain was Escherichia coli J53 $\left(\mathrm{Rif}^{\mathrm{R}}\right)$. Briefly, the donor and recipient strains were grown in Luria-Bertani medium overnight at $30{ }^{\circ} \mathrm{C}$, mixed in equal volumes, grown to mid-exponential phase $(20 \mathrm{~h})$, plated on Mueller-Hinton agar plates containing rifampicin $(100 \mu \mathrm{g}$ $\left.\mathrm{ml}^{-1}\right)$ and streptomycin $\left(20 \mu \mathrm{g} \mathrm{ml}^{-1}\right)$ and incubated for $24 \mathrm{~h}$ at $30{ }^{\circ} \mathrm{C}$. The transconjugants were identified phenotypically by API $20 \mathrm{E}$ and tested for the presence and size of the variable part of the transferred integrons by PCR analyses. MIC values of streptomycin and rifampicin of donor strains were determined according to a standard method.

Statistical analysis. The statistical significance between the susceptibility and number of integron-positive and integron-negative isolates was calculated using Pearson's $\chi^{2}$ test.

\section{RESULTS}

\section{Molecular identification of strains by hsp60 sequence analysis}

PCR amplification of part of the $h s p 60$ heat-shock protein gene resulted in a $341 \mathrm{bp}$ product for all strains. The PCR products were sequenced and $272 \mathrm{nt}$ sequences were compared with the sequences of type and reference strains of Enterobacter species. All strains phenotypically identified as E. cloacae were clustered within the E. cloacae complex. The majority of the strains were identified as E. hormaechei (84\%), with ' $E$. hormaechei subsp. steigerwaltii' being the most frequent subspecies $(69.6 \%)$. Five strains $(7.2 \%)$ were identified as ' $E$. hormaechei subsp. hormaechei'. Five isolates $(7.2 \%)$ belonged to ' $E$. hormaechei subsp. oharae', six $(8.7 \%)$ to E. cloacae cluster III and three to E. cloacae cluster IV. One strain was identified as E. cloacae subsp. cloacae and one as E. kobei. ERIC-PCR analysis showed that the strains were genetically unrelated (see Supplementary Fig. S1, available in JMM Online).

\section{Antimicrobial susceptibility}

We analysed the profiles of resistance to 15 antibiotics belonging to eight groups (Table 1). Five strains of ' $E$. hormaechei subsp. steigerwaltii' and two strains of 'E. hormaechei subsp. oharae' produced extended-spectrum $\beta$-lactamases. Fifty-two isolates $(75.4 \%)$ were resistant to more than three unrelated antibiotics. Four strains (5.8\%), belonging to 'E. hormaechei subsp. steigerwaltii' (three) and E. cloacae cluster IV (one), were resistant to all of the 15 antibiotics used in the test. Two of these strains were isolated from blood and two from urine. Altogether, $71.4 \%$ strains of ' $E$. hormaechei subsp. steigerwaltii' were multiresistant, $67 \%$ of $E$. cloacae cluster IV, $50 \%$ of E. cloacae cluster III and $40 \%$ of ' $E$. hormaechei subsp. hormaechei' and ' $E$. hormaechei subsp. oharae'. The percentage of multiresistant strains in hospital no. 1 was $64 \%$, whereas in hospital no. 2 it reached $54.5 \%$. The highest resistance percentages were found towards sulfamethoxazole (78.3\%), ticarcillin $(63.8 \%)$, ciprofloxacin $(64.6 \%)$, tetracycline $(62.3 \%)$, cefotaxime $(59.4 \%)$, amikacin $(56.5 \%)$, tobramycin $(56.5 \%)$, ceftazidime $(55.7 \%)$, netilmicin, gentamicin and co-trimoxazole $(55.1 \%)$, followed by piperacillin, piperacillin + tazobactam and chloramphenicol $(52.2 \%)$ and imipenem (14.5\%) (Table 1). The resistance to imipenem in all cases was conferred by an AmpC-mediated mechanism.

\section{Multiplex PCR analysis for identification of integron integrase genes}

Class 1 integrons were detected in 38 isolates (55.1\%). No intI2 and intI3 genes were found. Thirty-one strains $(63.3 \%)$ of ' $E$. hormaechei subsp. steigerwaltii' were intI1positive, three strains $(50 \%)$ of E. cloacae cluster III, two strains $(40 \%)$ of ' $E$. hormaechei subsp. oharae', one ( $33.3 \%$ ) belonging to E. cloacae cluster IV and one strain ( $20 \%)$ of ' $E$. hormaechei subsp. hormaechei. The resistance to each of the antibiotics, except imipenem, was significantly associated with the presence of integrons $(P<0.01)$ (Table 1). Moreover, of the 52 strains $(75.4 \%)$ resistant to more than three antibiotics, $76 \%$ carried an integron, whereas none of the susceptible strains or those resistant to only one of the antimicrobial agents revealed the presence of the intI gene. All of the 38 integron-positive strains were resistant to more than three antibiotics; in fact, $92.1 \%(35$ isolates) of the integron-positive strains were resistant to more than 10 antibiotics.

\section{Analysis of the variable region of class 1 integrons}

We detected and identified gene cassettes in 37/38 intIpositive strains. The variable part of the class 1 integron was amplified using primers complementary to the conserved regions of the platform flanking the inserted resistance genes. The variable region of $180 \mathrm{bp}$ present in the integron of an E. cloacae cluster IV strain indicated a structure with no incorporated cassettes. The other amplicon sizes were 1.0, 1.6 and $1.9 \mathrm{~kb}$ (Table 2). A $1.0 \mathrm{~kb}$ product was present in two integrons found in strains belonging to ' $E$. hormaechei subsp. oharae' and E. cloacae cluster III. A single amplicon of $1.6 \mathrm{~kb}$ was found in 10 strains belonging to ' $E$. hormaechei subsp. steigerwaltii' 
Table 1. Association between resistance to antibiotics and the presence of integrons in strains of the E. cloacae complex

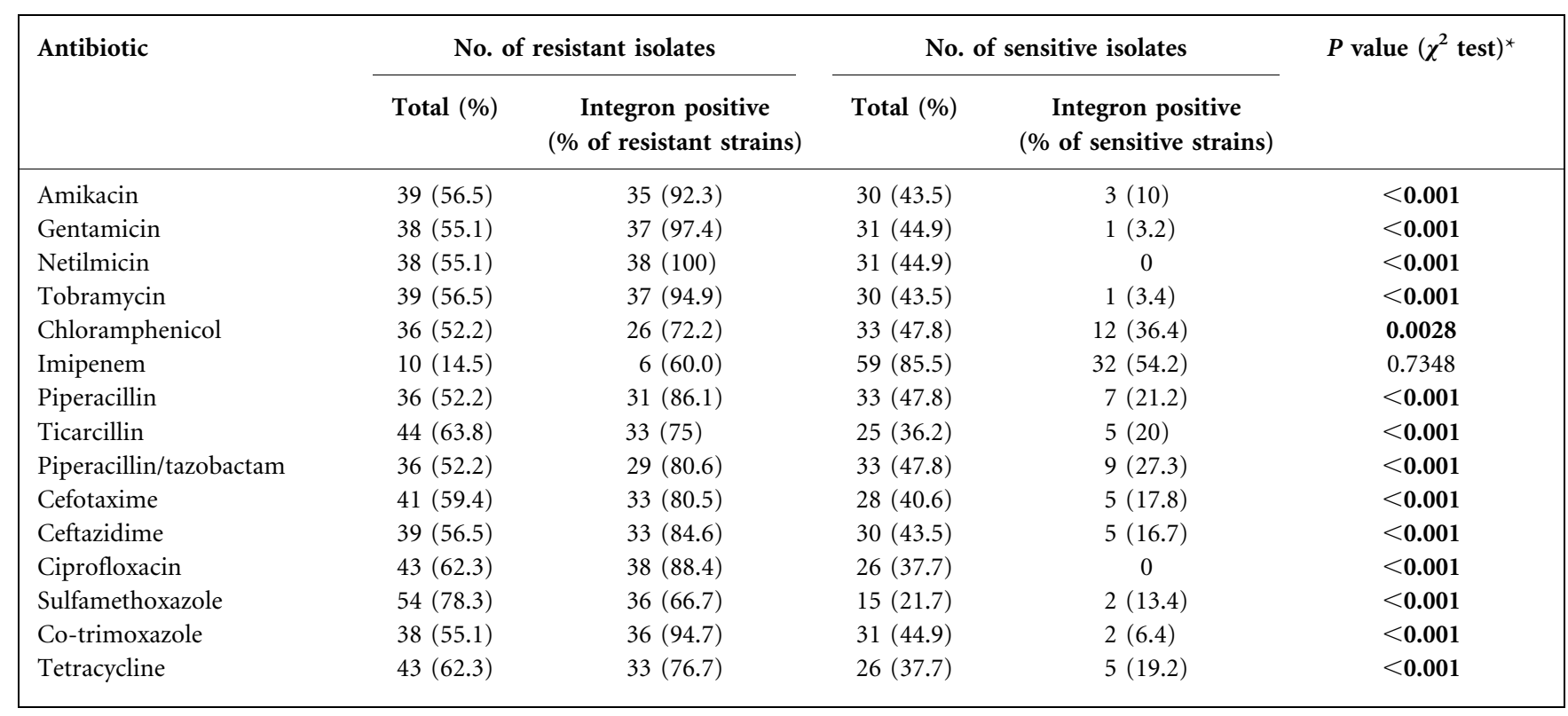

*Significant values are in bold.

(six), E. cloacae cluster III (two), 'E. hormaechei subsp. hormaechei' (one) and 'E. hormaechei subsp. oharae' (one). An amplicon of $1.9 \mathrm{~kb}$ was obtained for 16 strains of ' $E$. hormaechei subsp. steigerwaltii. Two amplicons of 1.6 and $1.9 \mathrm{~kb}$ were found exclusively in nine strains identified as 'E. hormaechei subsp. steigerwaltii', eight of which were isolated from urine and one from a catheter.

The sequence analysis identified two groups of genes: those encoding aminoglycoside adenylotransferase responsible for resistance to aminoglycosides, and $d f r$ cassettes conferring resistance to trimethoprim. Genes encoding aminoglycoside adenylotransferase represented $58.2 \%$ of all cassettes found and included aadA2 $(31.6 \%$ of all cassettes), aadA1 (15.2\%), both conferring resistance to streptomycin and spectinomycin, and $\operatorname{aadB}(10.1 \%)$ encoding aminoglycoside-2"-O-nucleotidyltransferase that confers resistance to amikacin, gentamicin, tobramycin, dibekacin, sisomicin, netilmicin and kanamycin. One strain of 'E. hormaechei subsp. steigerwaltii' had an aacA4 cassette $(1.3 \%$ of all cassettes) responsible for resistance to tobramycin and amikacin. The $d f r$ cassettes, representing $41.8 \%$ of all cassettes detected, included $d f r A 12(30.4 \%)$ and $d f r A 1(11.4 \%)$.

The cassette arrays were as follow: $d f r A 12$-orfF-aadA2 for the $1.9 \mathrm{~kb}$ CS-PCR product and aadB-aadA2, dfrA1-aadA1 and aacA4-aadA1 for the $1.6 \mathrm{~kb}$ CS-PCR products (Table 2).

Table 2. Distribution of gene cassette arrays among intl-positive strains of the E. cloacae complex within hospitals

\begin{tabular}{|c|c|c|c|}
\hline \multirow[t]{2}{*}{ Type of gene cassette array } & \multirow{2}{*}{$\begin{array}{c}\text { Size of integron } \\
\text { variable region }(\mathbf{k b})\end{array}$} & \multicolumn{2}{|c|}{ Nomenspecies and no. of strains with gene cassette array } \\
\hline & & Hospital 1 & Hospital 2 \\
\hline aadA1 & 1.0 & $\begin{array}{l}\text { 'E. hormaechei subsp. oharae' } \\
(1), \text { E. cloacae cluster III (1) }\end{array}$ & \\
\hline aadB-aadA2 & 1.6 & & $\begin{array}{l}\text { 'E. hormaechei subsp. steigerwaltii' (3), 'E. } \\
\text { hormaechei subsp. hormaechei' (1), E. } \\
\text { hormaechei subsp. oharae' (1), E. cloacae } \\
\text { cluster III (1) }\end{array}$ \\
\hline$d f r A 1-a a d A 1$ & 1.6 & $\begin{array}{l}\text { 'E. hormaechei subsp. steigerwaltii' } \\
(2), \text { E. cloacae cluster III (1) }\end{array}$ & \\
\hline aacA4-aadA1 & 1.6 & 'E. hormaechei subsp. steigerwaltii' (1) & \\
\hline dfrA12-orfF-aadA2 & 1.9 & 'E. hormaechei subsp. steigerwaltii' (1) & 'E. hormaechei subsp. steigerwaltii' (15) \\
\hline dfrA1-aadA1, dfrA12-orfF-aadA2 & $1.6 / 1.9$ & 'E. hormaechei subsp. steigerwaltii' (6) & \\
\hline aadB-aadA2, dfrA12-orfF-aadA2 & $1.6 / 1.9$ & & 'E. hormaechei subsp. steigerwaltii' (3) \\
\hline
\end{tabular}


The $d f r A 12-o r f F-a a d A 2$ array was present in strains from both hospitals. Hospital-dependent differences were observed for the $d f r A 1$-aadA1 array, which was found exclusively in the genomes of strains from the first hospital, and for the aadB-aadA2 array, identified only in strains from the other hospital. The cassette arrays of integrons identified in the E. cloacae complex together with the hospital affiliations are listed in Table 2. The gene cassette array found most often was dfrA12-orfF-aadA2 in the $1.9 \mathrm{~kb}$ variable region present exclusively in ' $E$. hormaechei subsp. steigerwaltii. The CLUSTAL W-based comparison of the sequences showed them to be identical for $>95 \%$, regardless of whether they were the only integron in the bacterial genome or a second one. This cassette array was found in strains from both hospitals.

\section{Conjugation assay}

Integrons were transferred to E. coli $\mathrm{J}-53\left(\mathrm{Rif}^{\mathrm{R}}\right)$ in a conjugation assay. Thirty-seven transconjugants were selected on media complemented with antibiotics and screened for the presence of intI1 and variable region size. All of the integrons were on transferable genetic elements, as we managed to produce transconjugants with integron platforms. Transconjugants were analysed for the acquired resistance. The transferred resistance primarily included that to aminoglycosides, ticarcillin, piperacillin, sulfamethoxazole, trimethoprim and tetracycline. Additionally, for selected strains with different integrons and resistance patterns, we performed plasmid analyses of donor and transconjugant isolates to check whether the integrons were embedded in transferable plasmids. We found plasmids present in both donor and transconjugant isolates.

\section{DISCUSSION}

The phenotypic similarity of strains belonging to different species of the E. cloacae complex creates many problems with their identification. We used sequencing of the $h s p 60$ gene, which shows good discriminatory power, to identify species and subspecies within the E. cloacae complex (Paauw et al., 2008). The majority of clinical strains were identified as $E$. hormaechei, with ' $E$. hormaechei subsp. steigerwaltii' as a predominant group. E. hormaechei strains were isolated from a variety of samples, including urine, wounds, blood and stools. However, strains of E. cloacae III and IV and E. cloacae subsp. cloacae clusters can also be pathogenic to humans, as they were isolated from nine infections.

Among the published reports, the resistance of E. cloacae strains varies depending on geographical location and year of testing (Sanders \& Sanders, 1997). We noted higher levels of resistance, described as a percentage of resistant strains, than those reported earlier (Paterson, 2006; Turner, 2008; Vakulenko \& Mobashery, 2003). Over $60 \%$ of strains were resistant to sulfamethoxazole, ticarcillin, ciprofloxacin and tetracycline, and more than $50 \%$ were resistant to aminoglycosides, cefotaxime, ceftazidime, co-trimoxazole, piperacillin, piperacillin + tazobactam and chloramphenicol. In the study performed under the European SENTRY programme, in reports from 2003, including over 7000 clinical isolates of four Enterobacteriaceae genera, the susceptibility to amikacin, gentamicin and tobramycin among Enterobacter species was 95.8, 90.1 and 82.1\%, respectively (Vakulenko \& Mobashery, 2003). In the same report, analysis of more than 4000 strains of Enterobacteriaceae in the USA revealed that Enterobacter species together with other enterobacterial genera are quite susceptible to aminoglycosides: $96-100 \%$ to amikacin, 90 $96 \%$ to gentamicin and $94-97 \%$ to tobramycin. Turner (2008) reported the results of the SENTRY programme from 2006 and its comparison with results from 2002. All antibiotics demonstrated a reduction in activity against Enterobacter species in 2006 in comparison with the results from 2002: the percentage of sensitive strains was $89.3 \%$ for gentamicin ( $94.5 \%$ in 2002), $86.7 \%$ (90.4\%) for tobramycin, $86.0 \%(91.9 \%)$ for ciprofloxacin, $75.2 \%$ $(76.4 \%)$ for ceftazidime, $74.3 \%$ (78\%) for piperacillin + tazobactam and $98.3 \%(98.7 \%)$ for imipenem.

The percentage of E. cloacae complex strains possessing integrons was also higher $(53.6 \%)$ in comparison with earlier studies (Daikos et al., 2007; Schmitz et al., 2001). All int-positive strains were multiresistant, i.e. resistant to more than three unrelated antimicrobials. Schmitz et al. (2001) compared the frequency of intI1-positive E. cloacae strains, which ranged from $9.5 \%$ in 1993 to $36.4 \%$ in 1999 , with the sizes of the variable part of the integron, ranging from $0.8 \mathrm{~kb}$ in 1993 to $1.5 \mathrm{~kb}$ in 1999. Daikos et al. (2007) found $44.4 \%$ of intI1-positive Enterobacter species isolated from bloodstream infections with variable regions of 1.0, $1.6,1.7$ and $3.2 \mathrm{~kb}$. In our research, most of the integrons $(76.3 \%)$ were carried by ' $E$. hormaechei subsp. steigerwaltii, a predominant group in infections caused by the $E$. cloacae complex. Moreover only 'E. hormaechei subsp. steigerwaltii' strains harboured two integrons and those with the largest variable region $(1.9 \mathrm{~kb})$.

Integron-positive strains demonstrated a greater tendency towards multiple antibiotic resistance than integronnegative isolates $(P<0.01)$. The resistance for each of the antibiotics, except for imipenem, was significantly associated with the presence of integrons $(P<0.01)$ (Table 1$)$. Moreover, of the 61 isolates $(88.4 \%)$ resistant to more than three antibiotics, $60.7 \%$ carried an integron, whereas none of the strains that were susceptible or resistant to one of the antimicrobial agents revealed the presence of the intI gene. All of the 38 integron-positive strains were resistant to more than three antibiotics; in fact, $94.6 \%$ of them (35 isolates) were resistant to more than 10 antibiotics, indicating that the presence of integrons in the E. cloacae complex strains is associated with multidrug resistance regardless of the gene cassette content $(P<0.01)$. Studies by Leverstein-van Hall et al. (2002b, 2003) showed that resistance is associated with the presence of integrons in members of the Enterobacteriaceae and that strains with 
integrons of class 1 are $100 \%$ resistant to more than one antibiotic. Moreover, horizontal gene transfer of integrons often occurs and is very efficient in a hospital environment, and the intra- and interspecies transfer of integrons plays a dominant role in development of multiresistance in the Enterobacteriaceae (Leverstein-van Hall et al., 2002a). We noted a high rate of integron-containing-element transfer in vitro and showed that the integrons were embedded in transferable plasmids. The transferred resistance was not limited to aminoglycosides, trimethoprim and sulfamethoxazole; it also included resistance to other classes of antimicrobials, e.g. $\beta$-lactams. Resistance genes were present on the same plasmid and possibly were part of an extended integron in which additional resistance genes are located after orf513 (Paauw et al., 2006; Sabaté et al., 2002).

Analyses of the variable part of the integrons showed only a limited diversity of gene cassettes. We noticed locationdependent differences in the type of gene cassette content; namely, $d f r A 1$-aadA1 was found exclusively in the genomes of strains from the first hospital, whereas aadB-aadA2 was found from the second hospital, regardless of whether they were the only one or the second integron in the genome. The gene cassettes identified conferred resistance to older antimicrobials seldom used therapeutically nowadays, namely trimethoprim and aminoglycosides such as spectinomycin and streptomycin. In the first hospital, in almost all integrons, the $d f r$ cassettes were nearer the 5 '-end of the integron, so they were expressed more efficiently. This also suggests that selection for cassettes carrying $d f r$ genes might have occurred in this population.

Comparison of sequences of $d f r A 12$-orfF-aadA2 cassette arrays with those available in GenBank showed that they were $>99 \%$ identical to integrons described in the Enterobacteriaceae, and also in non-fermenting rods and staphylococci isolated from both human and animal samples (Leverstein-van Hall et al., 2002a; Kadlec \& Schwarz, 2008).

The dfr1-aadA1 cassette array was present as a single integron in two strains of ' $E$. hormaechei subsp. steigerwaltii isolated from blood and E. cloacae cluster III originating from aspirate, and, as a second platform, in six isolates of 'E. hormaechei subsp. steigerwaltii'. Previously, this cassette has been shown in other members of the Enterobacteriaceae and in E. cloacae within a larger integron associated with $b l a_{\mathrm{VIM}}$ genes (Kang et al., 2004; Leverstein-van Hall et al., 2002a). The aadB-aadA2 array was found in nine strains: as a single integron in three ' $E$. hormaechei subsp. steigerwaltii', one ' $E$. hormaechei subsp. hormaechei', one ' $E$. hormaechei subsp. oharae' and one E. cloacae cluster III, and as a second platform in three ' $E$. hormaechei subsp. steigerwaltii'; they had a similar sequence ( $98 \%$ similarity) to that of E. cloacae isolated in Nanfang Hospital in China (GenBank accession no. GU906259.1).

One strain of 'E. hormaechei subsp. steigerwaltii' had an aacA4aadA1 cassette array with $97 \%$ identity to an Aeromonas caviae partial class 1 integron sequence containing the oxa10, aacA4 and aadA1 genes (GenBank accession no. FM207629.1).
In summary, we found a high frequency of multidrug resistance and integron carriage among the E. cloacae complex strains of human origin, especially ' $E$. hormaechei subsp. steigerwaltii. The types of gene cassettes and the gene arrays in the E. cloacae complex seemed to be protected and not liable to changes. Our assumption is that there is a tendency to gather the whole integron rather than to incorporate new gene cassettes. It seems that horizontal gene transfer is a phenomenon that is more prevalent than integration of gene cassettes by integron integrase.

\section{REFERENCES}

Andersson, D. I. \& Hughes, D. (2010). Antibiotic resistance and its cost: is it possible to reverse resistance? Nat Rev Microbiol 8, 260-271.

CLSI (2009). Performance Standards for Antimicrobial Disk Susceptibility Tests; Approved Standard, 10th edn. M02-A10. Wayne, PA: Clinical and Laboratory Standards Institute.

Coudron, P. E., Moland, E. S. \& Thomson, K. S. (2000). Occurrence and detection of AmpC $\beta$-lactamases among Escherichia coli, Klebsiella pneumoniae, and Proteus mirabilis isolates at a Veterans Medical Center. J Clin Microbiol 38, 1791-1796.

Daikos, G. L., Kosmidis, C., Tassios, P. T., Petrikkos, G., Vasilakopoulou, A., Psychogiou, M., Stefanou, I., Avlami, A. \& Katsilambros, N. (2007). Enterobacteriaceae bloodstream infections: presence of integrons, risk factors, and outcome. Antimicrob Agents Chemother 51, 2366-2372.

Dillon, B., Thomas, L., Mohmand, G., Zelynski, A. \& Iredell, J. (2005). Multiplex PCR for screening of integrons in bacterial lysates. J Microbiol Methods 62, 221-232.

Fluit, A. C. \& Schmitz, F. J. (2004). Resistance integrons and superintegrons. Clin Microbiol Infect 10, 272-288.

Fraser, S. L., Arnett, M. \& Sinave, C. P. (2008). Enterobacter infections. eMedicine http://emedicine.medscape.com/article/216845-overview.

Hall, R. M. \& Collis, C. M. (1995). Mobile gene cassettes and integrons: capture and spread of genes by site-specific recombination. Mol Microbiol 15, 593-600.

Hoffmann, H. \& Roggenkamp, A. (2003). Population genetics of the nomenspecies Enterobacter cloacae. Appl Environ Microbiol 69, 53065318.

Hoffmann, H., Stindl, S., Ludwig, W., Stumpf, A., Mehlen, A., Monget, D., Pierard, D., Ziesing, S., Heesemann, J. \& other authors (2005). Enterobacter hormaechei subsp. oharae subsp. nov., E. hormaechei subsp. hormaechei comb. nov., and E. hormaechei subsp. steigerwaltii subsp. nov., three new subspecies of clinical importance. J Clin Microbiol 43, 3297-3303.

Kadlec, K. \& Schwarz, S. (2008). Analysis and distribution of class 1 and class 2 integrons and associated gene cassettes among Escherichia coli isolates from swine, horses, cats and dogs collected in the BfT-GermVet monitoring study. J Antimicrob Chemother 62, 469473.

Kang, C.-I., Kim, S.-H., Park, W. B., Lee, K.-D., Kim, H.-B., Oh, M., Kim, E.-C. \& Choe, K.-W. (2004). Bloodstream infections caused by Enterobacter species: predictors of 30-day mortality rate and impact of broad-spectrum cephalosporin resistance on outcome. Clin Infect Dis 39, 812-818.

Lee, K., Chong, Y., Shin, H. B., Kim, Y. A., Yong, D. \& Yum, J. H. (2001). Modified Hodge and EDTA-disk synergy tests to screen metallo- $\beta$-lactamase-producing strains of Pseudomonas and Acinetobacter species. Clin Microbiol Infect 7, 88-91. 
Leverstein-van Hall, M. A., Box, A. T., Blok, H. E. M., Paauw, A., Fluit, A. C. \& Verhoef, J. (2002a). Evidence of extensive interspecies transfer of integron-mediated antimicrobial resistance genes among multidrug-resistant Enterobacteriaceae in a clinical setting. J Infect Dis 186, 49-56.

Leverstein-van Hall, M. A., Paauw, A., Box, A. T., Blok, H. E., Verhoef, J. \& Fluit, A. C. (2002b). Presence of integron-associated resistance in the community is widespread and contributes to multidrug resistance in the hospital. J Clin Microbiol 40, 3038-3040.

Leverstein-van Hall, M. A., Blok, H. E. M., Donders, A. R. T., Paauw, A., Fluit, A. C. \& Verhoef, J. (2003). Multidrug resistance among Enterobacteriaceae is strongly associated with the presence of integrons and is independent of species or isolate origin. J Infect Dis 187, 251259.

Lévesque, C., Piché, L., Larose, C. \& Roy, P. H. (1995). PCR mapping of integrons reveals several novel combinations of resistance genes. Antimicrob Agents Chemother 39, 185-191.

Mazel, D. (2006). Integrons: agents of bacterial evolution. Nat Rev Microbiol 4, 608-620.

Morand, P. C., Billoet, A., Rottman, M., Sivadon-Tardy, V., Eyrolle, L., Jeanne, L., Tazi, A., Anract, P., Courpied, J. P. \& other authors (2009). Specific distribution within the Enterobacter cloacae complex of strains isolated from infected orthopedic implants. J Clin Microbiol 47, 2489-2495.

Nijssen, S., Florijn, A., Top, J., Willems, R., Fluit, A. \& Bonten, M. (2005). Unnoticed spread of integron-carrying Enterobacteriaceae in intensive care units. Clin Infect Dis 41, 1-9.

Norrby, S. R. (2005). Integrons: adding another threat to the use of antibiotic therapy. Clin Infect Dis 41, 10-11.

Paauw, A., Fluit, A. C., Verhoef, J. \& Leverstein-van Hall, M. A. (2006). Enterobacter cloacae outbreak and emergence of quinolone resistance gene in Dutch hospital. Emerg Infect Dis 12, 807-812.

Paauw, A., Caspers, M. P. M., Schuren, F. H. J., Leverstein-van Hall, M. A., Delétoile, A., Montijn, R. C., Verhoef, J. \& Fluit, A. C. (2008).
Genomic diversity within the Enterobacter cloacae complex. PLoS ONE 3, e3018.

Paauw, A., Caspers, M. P. M., Leverstein-van Hall, M. A., Schuren, F. H. J., Montijn, R. C., Verhoef, J. \& Fluit, A. C. (2009). Identification of resistance and virulence factors in an epidemic Enterobacter hormaechei outbreak strain. Microbiology 155, 1478-1488.

Paterson, D. L. (2006). Resistance in Gram-negative bacteria: Enterobacteriaceae. Am J Med 119 (Suppl. 1), S20-S28, discussion S62-S70.

Sabaté, M., Navarro, F., Miró, E., Campoy, S., Mirelis, B., Barbé, J. \& Prats, G. (2002). Novel complex sul1-type integron in Escherichia coli

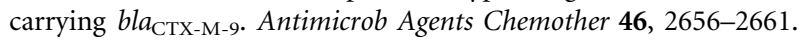

Sanders, W. E., Jr \& Sanders, C. C. (1997). Enterobacter spp.: pathogens poised to flourish at the turn of the century. Clin Microbiol Rev 10, 220-241.

Schmitz, F. J., Hafner, D., Geisel, R., Follmann, P., Kirschke, C., Verhoef, J., Köhrer, K. \& Fluit, A. C. (2001). Increased prevalence of class I integrons in Escherichia coli, Klebsiella species, and Enterobacter species isolates over a 7-year period in a German university hospital. J Clin Microbiol 39, 3724-3726.

Tsakris, A., Kristo, I., Poulou, A., Themeli-Digalaki, K., Ikonomidis, A., Petropoulou, D., Pournaras, S. \& Sofianou, D. (2009). Evaluation of boronic acid disk tests for differentiating KPC-possessing Klebsiella pneumoniae isolates in the clinical laboratory. J Clin Microbiol 47, 362367.

Turner, P. J. (2008). Meropenem activity against European isolates: report on the MYSTIC (Meropenem Yearly Susceptibility Test Information Collection) 2006 results. Diagn Microbiol Infect Dis 60, 185-192.

Vakulenko, S. B. \& Mobashery, S. (2003). Versatility of aminoglycosides and prospects for their future. Clin Microbiol Rev 16, 430-450.

Versalovic, J., Koeuth, T. \& Lupski, J. R. (1991). Distribution of repetitive DNA sequences in eubacteria and application to fingerprinting of bacterial genomes. Nucleic Acids Res 19, 6823-6831. 